\title{
Efficient quantum transport simulation for bulk graphene heterojunctions
}

\author{
Ming-Hao Liu (劉明豪) ${ }^{*}$ and Klaus Richter \\ Institut für Theoretische Physik, Universität Regensburg, D-93040 Regensburg, Germany
}

(Received 1 June 2012; revised manuscript received 10 August 2012; published 28 September 2012)

\begin{abstract}
The quantum transport formalism based on tight-binding models is known to be powerful in dealing with a wide range of open physical systems subject to external driving forces but is, at the same time, limited by the memory requirement's increasing with the number of atomic sites in the scattering region. Here we demonstrate how to achieve an accurate simulation of quantum transport feasible for experimentally sized bulk graphene heterojunctions at a strongly reduced computational cost. Without free tuning parameters, we show excellent agreement with a recent experiment on Klein backscattering [A. F. Young and P. Kim, Nature Phys. 5, 222 (2009)].
\end{abstract}

DOI: 10.1103/PhysRevB.86.115455

PACS number(s): 72.80.Vp, 72.10.Bg, 73.23.Ad, 73.40.Gk

\section{INTRODUCTION}

Electronic transport is one of the important fields among the increasing number of fundamental studies ${ }^{1,2}$ of graphene, a one-atom-thick carbon honeycomb lattice. ${ }^{3}$ Due to the gapless and chiral nature of its electronic structure, graphene exhibits energy dispersions linear in momentum, the transport carriers behave like massless Dirac fermions, and the properties based on Schrödinger wave mechanics in semiconductor physics have to be re-treated by Dirac-type physics in graphene. Tunneling across $p n$ and $p n p$ junctions is perhaps the most popular example that shows how different the charge carriers behave, compared to semiconductor heterostructures. By solving the Dirac equation, perfect transmission at normal incidence across a potential step ${ }^{4}$ as well as a potential barrier ${ }^{5}$ was shown for monolayer graphene. This mimicks the Klein paradox in quantum electrodynamics ${ }^{6}$ and was later referred to as Klein tunneling, ${ }^{7,8}$ which attracted both experimental ${ }^{9-16}$ and further theoretical ${ }^{13,17-24}$ investigations.

The Dirac theory, an effective approach valid only for low-energy excitations, generally serves as a starting point for theoretical studies of transport in graphene and can often provide analytical results to capture basic physical insights for certain problems with simplified system geometries. For further considerations, such as to maintain the lattice information on graphene or to account for complicated geometries and more realistic factors, one has to resort to more advanced theoretical models. The tight-binding model (TBM), a commonly used semiemperical approach for electronic structure calculations in solid-state physics, ${ }^{25}$ allows for consideration of more complete band information on graphene at a low computational cost. The combination of the TBM with nonequilibrium Green's function approaches forms the modern quantum transport formalism, ${ }^{26}$ which is able to deal with a wide range of conductors composed of a scattering region and external leads with or without bias. The description of the graphene scattering region of interest, however, requires a TBM Hamiltonian matrix,

$$
\mathcal{H}_{\mathrm{gnr}}\left(V, t, t^{\prime}\right)=\sum_{n=1}^{N} V_{n} c_{n}^{\dagger} c_{n}-t \sum_{\langle m, n\rangle} c_{n}^{\dagger} c_{m}-t^{\prime} \sum_{\langle m, n\rangle\rangle} c_{n}^{\dagger} c_{m},
$$

whose matrix size depends on the involved number of atomic sites $N$ and therefore imposes a computational limit when addressing realistic experimental system sizes. This is partly the reason why many quantum transport studies address graphene "nanoribbons" rather than large-area graphene. The notation in Eq. (1) is described as follows: $t\left(t^{\prime}\right)$ is the
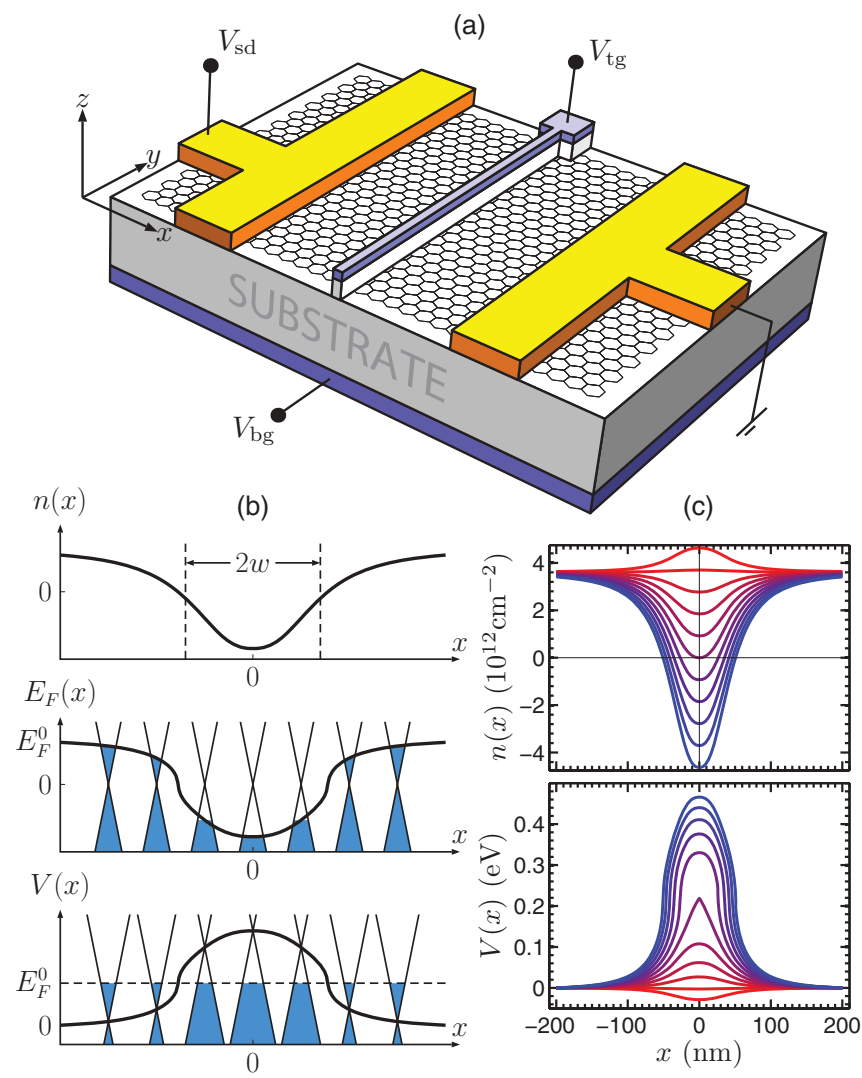

FIG. 1. (Color online) (a) Schematic of double-gated graphene. (b) Carrier density profile $n(x)$ (top) and its corresponding local Fermi level $E_{F}(x)$ (middle). The extracted potential profile $V(x)$ (bottom) is given by the difference between the global Fermi level $E_{F}^{0}$ and $E_{F}(x)$; see text. (c) Reproduced densities $n(x)$ provided in the Supplementary Material for Ref. 15, with $V_{\mathrm{bg}}=50 \mathrm{~V}$ and $V_{\mathrm{tg}}=-8.9,-7.9, \ldots, 0.1,1.1 \mathrm{~V}$ (curves from bottom to top), and the extracted corresponding $V(x)$ (curves from top to bottom). 
nearest (next-nearest)-neighbor hopping parameter, $V_{n}$ is the local potential energy at site $n, c_{n}^{\dagger}\left(c_{n}\right)$ creates (annihilates) a charge carrier at the $n$th site, and the summation $\sum_{\langle m, n\rangle}\left(\sum_{\langle\langle m, n\rangle\rangle}\right)$ runs over all $m$ and $n$ site indices that are nearest (next nearest) to each other within the scattering region.

Typical sizes of graphene flakes for experimental transport investigations amount to a few microns by a few microns, but even a $1 \mu \mathrm{m} \times 1 \mu \mathrm{m}$ graphene flake contains roughly $10^{7}$ atoms, leading to a spinless single-orbital TBM Hamiltonian matrix of more than $10^{14}$ elements that requires an exceeding memory and hence an unreasonable computation burden. TBM-based quantum transport for bulk materials therefore requires further improvements to overcome the issue of the limited scattering region size. In this paper, we demonstrate how an accurate TBM-based transport calculation for bulk graphene heterojunctions can be performed without free parameters, circumventing the problem of large system scales.

To achieve such a TBM bulk transport simulation, two crucial concepts are required, namely, extraction of a realistic potential profile and description of a bulk graphene scattering region, which are described in Sec. II, where a brief summary of the quantum transport formalism is also included (Sec. II C). In Sec. III, we revisit and simulate the recent Klein backscattering experiment ${ }^{15}$ for transport through doublegated graphene [as depicted in Fig. 1(a)] to compare with and to demonstrate our approach. Section IV summarizes the present work.

\section{THEORETICAL FORMULATION}

\section{A. Extraction of a realistic potential profile}

A theoretical study of transport in graphene, whether based on Dirac theory or the TBM formalism, requires the potential $V(x)$ as an input, which actually means the local energy offset of the Dirac point and is often regarded directly as the electric potential. In fact, the application of a gate voltage $V_{g}$ does not directly raise the Dirac cone by $-e V_{g}$ ( $-e$ being the electron charge) but enhances or depletes the carrier density, hence raising or lowering the local Fermi level. For double-gated graphene [Fig. 1(a)], the combination of a top-gate voltage $V_{\text {tg }}$ and a back-gate voltage $V_{\text {bg }}$ results in a carrier density profile $n(x)$ such as that shown in the upper panel in Fig. 1(b). Its energy dependence, $n(E)=$ $\operatorname{sgn}(E) E^{2} /\left[\pi\left(\hbar v_{F}\right)^{2}\right]$, is obtained by integrating the density of states over energy. Defining the local Fermi level as

$$
E_{F}(x)=\operatorname{sgn}[n(x)] \hbar v_{F} \sqrt{\pi|n(x)|},
$$

one obtains the spatially varying height of the filled states, as depicted in the middle panel in Fig. 1(b). In a transport calculation, the global Fermi level $E_{F}^{0}$ is a fixed quantity. Hence to account for the profiles of $E_{F}(x)$ and $n(x)$, one shifts the local band offset by applying a local potential,

$$
V(x)=E_{F}^{0}-E_{F}(x),
$$

as depicted in the lower panel in Fig. 1(b). This completes the extraction of the potential profile from the carrier density profile. Note that the above model makes use of the linear density of states that is normally valid in the experimental range of the carrier density, although the energy dispersion based on the TBM covers the full range. The energy range beyond the Dirac model with a nonlinear density of states can, in principle, be treated within the TBM similarly to the process introduced above, but this would be relevant only far from the energy range of interest.

A realistic carrier density profile depends on the experimental geometry and dielectric material of the gate fabrication. In the experiment in Ref. $15, n(x)$ was obtained from an electrostatic simulation and empirically described by

$$
n(x)=\left(\frac{12.8 V_{\mathrm{tg}}}{1+|x / w|^{2.5}}+V_{\mathrm{bg}}\right) C_{\mathrm{bg}},
$$

where 12.8 accounts for the effectiveness of the top gate relative to the back gate, $C_{\mathrm{bg}} \approx 7.23 \times 10^{10} \mathrm{~cm}^{-2} / \mathrm{V}$ is the classical (electron number) capacitance of a 290 -nm-thick $\mathrm{SiO}_{2}$ substrate, and the effective half-width of the top gate is $w=46 \mathrm{~nm} .{ }^{15}$ Figure 1 (c) shows various carrier density profiles described by Eq. (4), subject to $V_{\mathrm{bg}}=50 \mathrm{~V}$ and various $V_{\mathrm{tg}}$, and the extracted potential profiles, Eqs. (2) and (3).

\section{B. Bulk graphene scattering region}

In band theory, the electronic structure of a crystal lattice can be solved by applying the Bloch theorem, which allows us to reduce the problem with infinitely repeated unit cells to only one due to translation invariance along each space dimension. For transport calculations, however, the scattering region of interest is composed of a certain finite-size area and is generally not translationally invariant. For a large flake of double-gated graphene, such as that sketched in Fig. 1(a), the transverse dimension (along $y$ ) is typically a few microns in width so that the edges are of minor importance, and we can then assume translational invariance in the $y$ direction.

Consider bulk graphene oriented with zigzag carbon chains along the $x$ direction. Up to nearest-neighbor hopping, the minimal unit cell can be chosen as one hexagon row, i.e., a graphene nanoribbon with zigzag chain number $N_{z}=2$ with transverse periodicity $W=3 a, a \approx 1.42 \AA$ being the bond length. The wave function at the bottom site $\left\langle x, y_{B} \mid \varphi\right\rangle$ of the unit cell is related to that at the top site $\left\langle x, y_{T} \mid \varphi\right\rangle$ through the Bloch theorem as ${ }^{28}\left\langle x, y_{T}+a \mid \varphi\right\rangle=e^{i k_{y} W}\left\langle x, y_{B} \mid \varphi\right\rangle$, implying $\left|x, y_{T}\right\rangle\left\langle x, y_{T}+a\left|=e^{i k_{y} W}\right| x, y_{T}\right\rangle\left\langle x, y_{B}\right|$, where $k_{y}$ is the Bloch momentum defined within $k_{y} W \in[-\pi, \pi]$. This means that a kinetic hopping across the upper boundary of the unit cell $\left|x, y_{T}\right\rangle\left\langle x, y_{T}+a\right|$ can be equivalently expressed as a periodic hopping $\left|x, y_{T}\right\rangle\left\langle x, y_{B}\right|$ modulated by the phase $e^{i k_{y} W}$ arising from the Bloch theorem. Similarly, one can obtain for the lower boundary $\left|x, y_{B}\right\rangle\left\langle x, y_{B}-a\left|=e^{-i k_{y} W}\right| x, y_{B}\right\rangle\left\langle x, y_{T}\right|$. Incorporating these periodic hopping terms, the TBM Hamiltonian for a bulk graphene scattering region can therefore be written as

$$
\begin{aligned}
\mathcal{H}_{\text {bulk }}\left(V, t ; k_{y}\right)= & \mathcal{H}_{\mathrm{gnr}}(V, t, 0) \\
& +\left(-t e^{i k_{y} W} \sum_{m} c_{T_{m}}^{\dagger} c_{B_{m}}+\text { H.c. }\right),
\end{aligned}
$$

where $c_{T_{m}}^{\dagger}\left(c_{B_{m}}\right)$ creates (annihilates) a charge carrier at the top (bottom) edge site of the $m$ th hexagon along $x$, and 
$\mathcal{H}_{\text {gnr }}(V, t, 0)$, given in Eq. (1), describes an $N_{z}=2$ graphene nanoribbon. Note that the above description for a bulk scattering region is restricted neither to nearest-neighbor hopping $\left(t^{\prime}=0\right)$ nor to the material graphene. For the present bulk transport simulation, however, next-nearest-neighbor hopping does not play an important role and we adopt $t=3 \mathrm{eV}$ and $t^{\prime}=0$ throughout Sec. III.

\section{Quantum transport formalism}

The quantum transport simulation in the present work is restricted to the linear response regime at zero temperature. Thus the Landauer conductance

$$
g\left(E_{F}^{0}\right)=\frac{e^{2} / h}{2 k_{F}} \int_{-k_{F}}^{k_{F}} T\left(E_{F}^{0} ; k_{y}\right) d k_{y}
$$

is the main object and is obtained by integrating the transmission function

$$
T\left(E ; k_{y}\right)=\operatorname{Tr}\left(\Gamma_{R} G_{R} \Gamma_{L} G_{R}^{\dagger}\right),
$$

which is equivalent to the Fisher-Lee relation. ${ }^{29}$ The Fermi wave vector in Eq. (6) is approximated from the low-energy linear dispersion by $k_{F}=E_{F}^{0} /\left(\hbar v_{F}\right)=E_{F}^{0} /(3 t a / 2)$. Note that the spin degeneracy is neglected here, while the valley degeneracy is inherently incorporated in $\mathcal{H}_{\text {bulk }}$.

The retarded Green's function $G_{R}$ of the scattering region at energy $E$ in Eq. (7) is obtained from

$$
G_{R}\left(E ; k_{y}\right)=\frac{1}{E-\left[\mathcal{H}_{\text {bulk }}\left(V, t ; k_{y}\right)+\Sigma_{L}+\Sigma_{R}\right]},
$$

where $\mathcal{H}_{\text {bulk }}\left(V, t ; k_{y}\right)$ has been given in Eq. (5) and $\Sigma_{L}\left(\Sigma_{R}\right)$ is the self-energy due to the left (right) lead composed of a semi-infinite repetition of unit cells. Adopting a Schurdecomposition-based algorithm for the singular hopping matrix type, ${ }^{28}$ the periodic hoppings as used in $\mathcal{H}_{\text {bulk }}$ can also be included in $\Sigma_{L}$ and $\Sigma_{R}$, enabling us to study pure bulk-to-bulk transmission. The spectral matrix functions $\Gamma_{l}$, with $l=L, R$, in Eq. (7) are given by $\Gamma_{l}=i\left(\Sigma_{l}-\Sigma_{l}^{\dagger}\right)$.

\section{KLEIN BACKSCATTERING EXPERIMENT REVISITED}

\section{A. Gate-voltage dependence}

Now we revisit the experiment in Ref. 15 by considering the extracted realistic potential $V(x)$ and applying the bulk TBM transport formalism introduced above. As shown in Fig. 1(c), the potential profile saturates at roughly $\pm 200 \mathrm{~nm}$, so we consider a scattering region described by $\mathcal{H}_{\text {bulk }}\left(V(x), t ; k_{y}\right)$ with length $L_{x}=400 \mathrm{~nm}$. The transport is solely supported by the states at the global Fermi level, which is set to $E_{F}^{0}=E_{F}(x= \pm 200 \mathrm{~nm})$. We first investigate the top-gate voltage dependence of the single-mode conductance $g$. In Fig. 2(a), we directly compare the oscillating features of our computed $g$ with the experimental data $G_{\mathrm{YK}},{ }^{27}$ choosing the measured $G_{\mathrm{YK}}\left(V_{\mathrm{tg}}, V_{\mathrm{bg}}=40 \mathrm{~V}\right)$ and $G_{\mathrm{YK}}\left(V_{\mathrm{tg}}, V_{\mathrm{bg}}=60 \mathrm{~V}\right)$ curves as explicit examples. In both cases, the general features of the measured oscillating conductance are well captured by our TBM calculation. The Dirac point position of the locally gated region corresponds to the conductance dip. To the left of this minimum the transport is in the npn regime exhibiting Fabry-Pérot-type oscillations due to interference of backscattered waves between the $n p$ and the $p n$ interfaces. To the right of the dip, the transport enters the $n n^{\prime} n$ regime, where graphene becomes much more transparent than for $n p n$, resulting in the suppression of the interference and the rise in the conductance. This conductance asymmetry ${ }^{9,14,19,30}$ is the first indirect feature of Klein tunneling, which results in the decay of the transmission with the incident angle in the $n p$ (a)

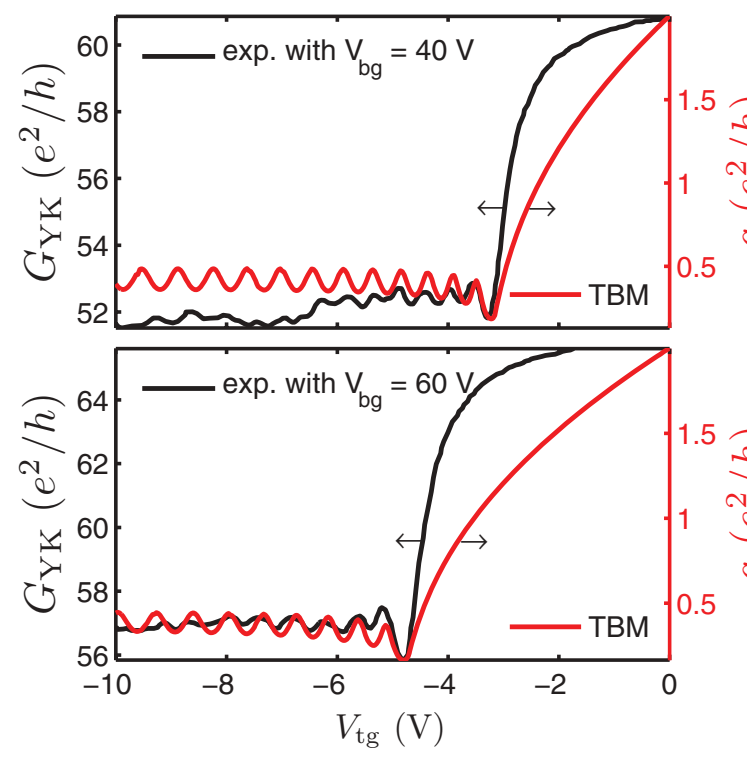

(b)

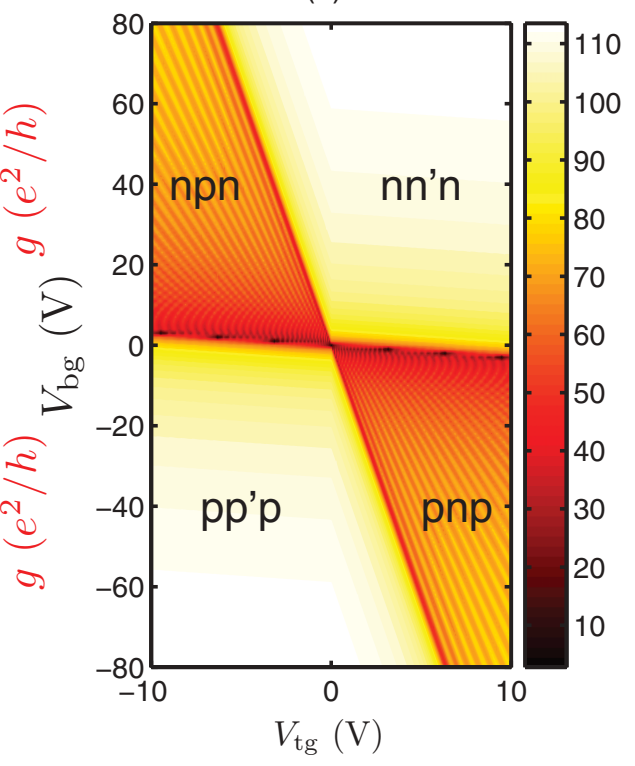

FIG. 2. (Color online) (a) Comparison of the top-gate voltage dependence of the measured conductance $G_{\mathrm{YK}}{ }^{27}$ and the computed single-mode conductance $g$ at $V_{\mathrm{bg}}=40 \mathrm{~V}$ and $V_{\mathrm{bg}}=60 \mathrm{~V}$. (b) Conductance map of $G\left(V_{\mathrm{tg}}, V_{\mathrm{bg}}\right)$. 
regime $^{4}$ and hence a lower integrated conductance, although the tunneling at normal incidence is perfect.

The single-mode spin-degenerate conductance $g$ from Eq. (6) has a maximum of $2 e^{2} / h$ and does not reflect the main effect of the back-gate voltage that tunes the global Fermi level $E_{F}^{0}$ : the modulation of the number of modes $M$ participating in transport. For bulk graphene at low energy, $M$ can be approximated by $2 k_{F} / \Delta k_{y}$ with $\Delta k_{y}=2 \pi / L_{y}$, where $L_{y}$ is the width of the graphene flake. This gives $M(E)=2 L_{y}|E| /\left(\pi \hbar v_{F}\right)$. While the calculation considers the bulk transport across the locally gated region in graphene, the contact resistance $R_{c}$ between the electrodes and graphene is not included. To compare with the full map of the measured $G_{\mathrm{YK}}\left(V_{\mathrm{tg}}, V_{\mathrm{bg}}\right)$, we temporarily adopt a simple model to account for multiple modes and contact resistance: $G\left(E_{F}^{0}\right)=\left\{\left[M\left(E_{F}^{0}\right) g\left(E_{F}^{0}\right)\right]^{-1}+R_{c}\right\}^{-1}$. Assuming an effective width $L_{y}=2 \mu \mathrm{m}$ and a low contact resistance, $R_{c}=0.2 \mathrm{k} \Omega$, we display the calculated top- and back-gate dependencies of $G\left(E_{F}^{0}\right)$ in Fig. 2(b), which qualitatively agrees with Ref. 15. Note that the quadrants of $G\left(V_{\mathrm{tg}}, V_{\mathrm{bg}}\right)$ are determined by the dependence of the potential profile on $V_{\mathrm{tg}}$ and $V_{\mathrm{bg}}$ and do not significantly change with the temporarily introduced parameters $L_{y}$ and $R_{c}$, on which we place less stress in the present work.

\section{B. Low-field magnetotransport}

Finally, we come to a closer analysis of the low-field magnetotransport. For an incoherent graphene pnp junction a perpendicular magnetic field leads to the increase in the magnetoresistance due to the bending of the electron trajectories. ${ }^{4}$ When the top gate is narrow enough, such as that in Ref. 15, with a width of about $20 \mathrm{~nm}$, a coherent graphene $p n p$ junction can be formed. Shytov et al. ${ }^{18}$ proposed a clever way to experimentally test the existence of Klein tunneling, making use of the sign change of the Klein backscattering phase at a weak magnetic field, which in turn results in a half-period shift of the Fabry-Pérot oscillations. Based on this semiclassical treatment the low-field magnetotransport experiment in Ref. 15 was regarded as providing evidence of Klein tunneling. In the following we show that our tuning-parameter-free TBM calculation confirms the semiclassical picture and, again, agrees well with the measurement.

The orbital contribution of the external magnetic field $B_{z}$ perpendicular to the graphene plane is incorporated in the TBM calculation through the Peierls substitution, ${ }^{31}$ while the Zeeman term is neglected since the Zeeman splitting is rather small compared to $E_{F}^{0}{ }^{2}$. To maintain the transverse (y) translation invariance throughout the whole system while also keeping the longitudinal $(x)$ translation invariance in the leads, we consider the Landau gauge of $\mathbf{A}=\left(0, x B_{z}, 0\right)$ only in the scattering region. Inside the left and right leads, however, constant gauge field strengths $A_{y}^{L}=x_{L} B_{z}$ and $A_{y}^{R}=x_{R} B_{z}$ must be considered, respectively, where $x_{L}$ and $x_{R}$ are the position coordinates of the leftmost and rightmost atomic site of the scattering region, in order to avoid a discontinuity of the vector potential.

Since the expected phase shift stems from Klein backscattering between the two interfaces inside the locally gated region, the potential tail does not play a crucial role and

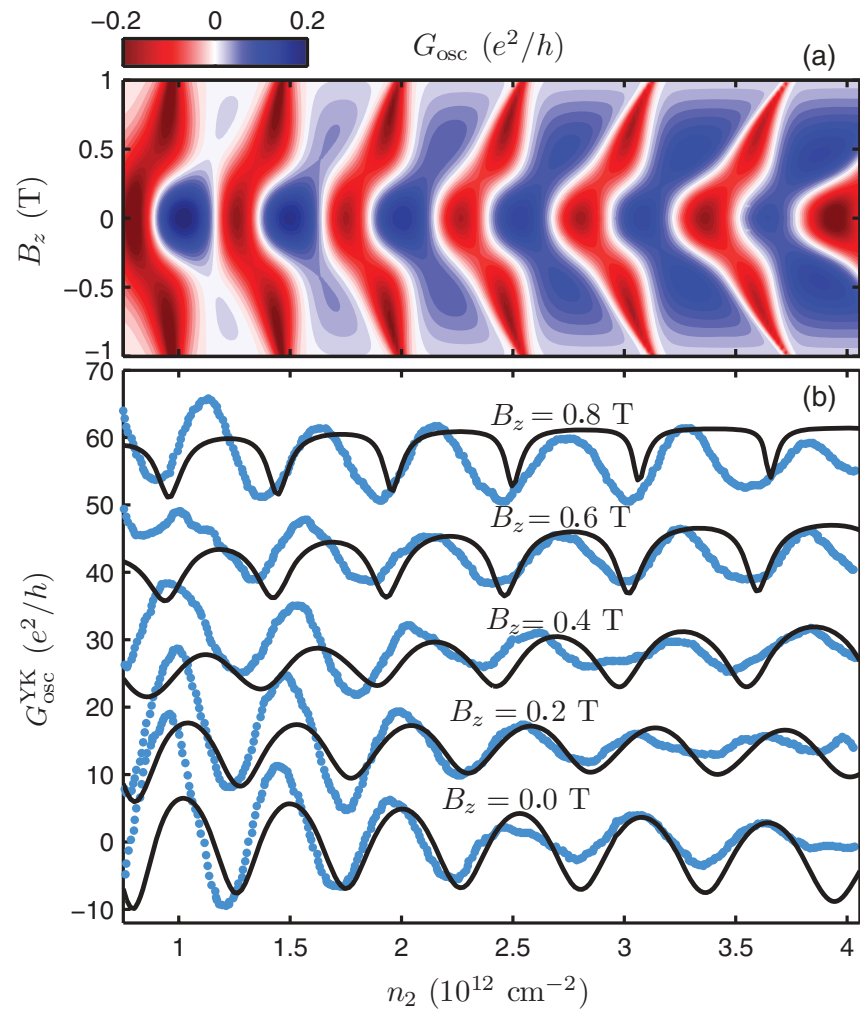

FIG. 3. (Color online) (a) Oscillating part of the computed conductance $G_{\text {osc }}\left(n_{2}, B_{z}\right)$ (see text for definition) as a function of the carrier density of the locally gated region $n_{2} \equiv n(x=0)$ and the external magnetic field $B_{z}$. (b) Comparison of computed $G_{\text {osc }}$ curves [solid (black) curves] at various magnetic field strengths with the experimental data from Ref. 15 [dotted gray (blue) curves].

we reduce the scattering region length to $L_{x}=150 \mathrm{~nm}$. Following the definition of the oscillating part of the conductance given in Ref. 15, we process our data on the single-mode conductance $g$ by first computing the odd part of the conductance, $G_{\text {odd }}\left(n_{2}, B_{z}\right)=g\left(n_{2}, B_{z}\right)-g\left(-n_{2}, B_{z}\right)$, and then subtracting its mean value to obtain $G_{\text {osc }}\left(n_{2}, B_{z}\right)=$ $G_{\text {odd }}\left(n_{2}, B_{z}\right)-\overline{G_{\text {odd }}\left(n_{2}, B_{z}\right)}$. Here $n_{2}=n(x=0)$ [see Eq. (4)] is the carrier density of the locally gated region. The obtained oscillation fringes of $G_{\text {osc }}\left(n_{2}, B_{z}\right)$ are shown in Fig. 3(a), which is, again, qualitatively consistent with Ref. 15 . The sudden phase shift, which indicates the presence of perfect transmission and corresponds to the half-period shift predicted by Shytov et al., ${ }^{18}$ occurs at magnetic field strengths between 0.2 and $0.4 \mathrm{~T}$ and is in excellent agreement with Ref. 15 . In Fig. 3(b), the computed $G_{\text {osc }}$ is compared with the experimental data $G_{\mathrm{osc}}^{\mathrm{YK}}\left(n_{2}, B_{z}\right)^{27}$ at various magnetic field strengths (both with offset for clarity).

\section{SUMMARY}

In conclusion, we have demonstrated the applicability of TBM-based quantum transport simulations for transport in bulk graphene heterojunctions. Applying the Bloch theorem along the transverse dimension, the computational effort for TBM transport through a bulk scattering region is significantly reduced. Together with the realistic potential profile extracted from the carrier density profile of a graphene $p n p$ junction, 
this method provides a confirmation of the experiment in Ref. 15 and its semiclassical theoretical interpretation, at a low computational cost without using free tuning parameters. The quantum transport approach presented here for studying bulk properties is suitable not only for graphene but also for other materials where the TBM works well.

\section{ACKNOWLEDGMENTS}

We appreciate valuable discussions with A. Cresti and V. Krueckl. Financial support from the Alexander von Humboldt Foundation (M.-H.L.) and Deutsche Forschungsgemeinschaft within GRK1570 (K.R.) is gratefully acknowledged.
*Corresponding author: minghao.liu.taiwan@gmail.com

${ }^{1}$ A. H. Castro Neto, F. Guinea, N. M. R. Peres, K. S. Novoselov, and A. K. Geim, Rev. Mod. Phys. 81, 109 (2009).

${ }^{2}$ S. Das Sarma, S. Adam, E. H. Hwang, and E. Rossi, Rev. Mod. Phys. 83, 407 (2011).

${ }^{3}$ K. S. Novoselov, A. K. Geim, S. V. Morozov, D. Jiang, Y. Zhang, S. V. Dubonos, I. V. Grigorieva, and A. A. Firsov, Science 306, 666 (2004).

${ }^{4}$ V. V. Cheianov and V. I. Fal'ko, Phys. Rev. B 74, 041403 (2006).

${ }^{5}$ M. I. Katsnelson, K. S. Novoselov, and A. K. Geim, Nat. Phys. 2 , 620 (2006).

${ }^{6}$ O. Klein, Z. Phys. 53, 157 (1929).

${ }^{7}$ C. W. J. Beenakker, Rev. Mod. Phys. 80, 1337 (2008).

${ }^{8}$ P. Allain and J. Fuchs, Eur. Phys. J. B 83, 301 (2011).

${ }^{9}$ B. Huard, J. A. Sulpizio, N. Stander, K. Todd, B. Yang, and

D. Goldhaber-Gordon, Phys. Rev. Lett. 98, 236803 (2007).

${ }^{10}$ J. R. Williams, L. DiCarlo, and C. M. Marcus, Science 317, 638 (2007).

${ }^{11}$ B. Özyilmaz, P. Jarillo-Herrero, D. Efetov, D. A. Abanin, L. S. Levitov, and P. Kim, Phys. Rev. Lett. 99, 166804 (2007).

${ }^{12}$ G. Liu, J. J. Velasco, W. Bao, and C. N. Lau, Appl. Phys. Lett. 92, 203103 (2008).

${ }^{13}$ R. V. Gorbachev, A. S. Mayorov, A. K. Savchenko, D. W. Horsell, and F. Guinea, Nano Lett. 8, 1995 (2008).

${ }^{14}$ N. Stander, B. Huard, and D. Goldhaber-Gordon, Phys. Rev. Lett. 102, 026807 (2009).

${ }^{15}$ A. F. Young and P. Kim, Nat. Phys. 5, 222 (2009).
${ }^{16}$ S.-G. Nam, D.-K. Ki, J. W. Park, Y. Kim, J. S. Kim, and H.-J. Lee, Nanotechnology 22, 415203 (2011).

${ }^{17}$ L. M. Zhang and M. M. Fogler, Phys. Rev. Lett. 100, 116804 (2008).

${ }^{18}$ A. V. Shytov, M. S. Rudner, and L. S. Levitov, Phys. Rev. Lett. 101, 156804 (2008).

${ }^{19}$ T. Low, S. Hong, J. Appenzeller, S. Datta, and M. S. Lundstrom, IEEE Trans. Electron Devices 56, 1292 (2009).

${ }^{20}$ A. Yamakage, K. I. Imura, J. Cayssol, and Y. Kuramoto, Europhys. Lett. 87, 47005 (2009).

${ }^{21}$ T. Low and J. Appenzeller, Phys. Rev. B 80, 155406 (2009).

${ }^{22}$ E. Rossi, J. H. Bardarson, P. W. Brouwer, and S. Das Sarma, Phys. Rev. B 81, 121408 (2010).

${ }^{23}$ M. Ramezani Masir, P. Vasilopoulos, and F. M. Peeters, Phys. Rev. B 82, 115417 (2010).

${ }^{24}$ M.-H. Liu, J. Bundesmann, and K. Richter, Phys. Rev. B 85, 085406 (2012).

${ }^{25}$ G. Grosso and G. P. Parravicini, Solid State Physics (Academic Press, New York, 2000).

${ }^{26}$ S. Datta, Electronic Transport in Mesoscopic Systems (Cambridge University Press, Cambridge, 1995).

${ }^{27}$ The experimental data compared in this work were extracted from the electronic file of Ref. 15, instead of the original data.

${ }^{28}$ M. Wimmer, Ph.D. thesis, Universität Regensburg, 2008.

${ }^{29}$ D. S. Fisher and P. A. Lee, Phys. Rev. B 23, 6851 (1981).

${ }^{30}$ J. Cayssol, B. Huard, and D. Goldhaber-Gordon, Phys. Rev. B 79, 075428 (2009).

${ }^{31}$ R. Peierls, Z. Phys. A 80, 763 (1933). 